\title{
Study of catheter associated urinary tract infection and biofilm production by Escherichia coli
}

\author{
K. Vidyasagar ${ }^{1, *}$, T. Nagarathnamma ${ }^{2}$ \\ ${ }^{\mathbf{1}}$ Assistant Professor, Dept. of Microbiology, Adichunchanagiri Institute of Medical Sciences, BG Nagara, Karnataka, ${ }^{\mathbf{P}}$ Professor, \\ BMC \& RI, Bangalore, Karnataka, India \\ *Corresponding Author: \\ Email: drsagar81@gmail.com
}

Received: $28^{\text {th }}$ March, 2018

Accepted: $18^{\text {th }}$ June, 2018

\begin{abstract}
Introduction: Catheter associated urinary tract infection (CAUTI) is most common nosocomial infection accounting for $40 \%$ of all institutionally acquired infections and is significantly associated with increased morbidity and health care costs. The study was undertaken to identify the incidence of CAUTI, the causative organisms and their antibiotic sensitivity and biofilm production by Escherichia coli in patients with urinary catheter for more than 24 hours.

Materials and Methods: The study included 250 hospitalized patients who catheterized for more than 24 hours. The urine sample aspirated from the port of catheter and subjected to wet mount and Gram staining and inoculated on blood agar, MacConkey agar and CLED media by standard loop method. The isolates with significant counts $\left(\geq 10^{2} \mathrm{CFU} / \mathrm{ml}\right)$ were identified and antibiotic susceptibility testing was done by Kirby Bauer disc diffusion methods as per CLSI guidelines. Escherichia coli isolates were tested for biofilm production qualitatively by Tube method and Congo Red Agar method.

Results: The present study showed CAUTI in $27.2 \%$ of the catheterized patients. Escherichia coli was the most common organism isolated (33.8\%). The most effective antibiotics against Gram negative isolates were Imipenem (95.23\%) and against Gram positive isolates were Vancomycin and Linezolid (100\% each). 91.6\% Escherichia coli isolates were biofilm producers.

Conclusion: The Study showed that the duration of catheterization ( $\geq 6$ days) is the most important predisposing factor for developing CAUTI. Escherichia coli is the most common organism causing CAUTI. The high incidence of resistance to conventional antibiotic such as Ampicillin, Co-trimoxazole and Ciprofloxacin among the pathogens causing CAUTI.
\end{abstract}

Keywords: CAUTI, Escherichia coli, Biofilm.

\section{Introduction}

Urinary tract infection poses a serious health threat with respect to antibiotic resistance and high recurrence rate. ${ }^{1}$ Urinary tract Infection (UTI) represents one of the most common diseases encountered in medical practice today whether hospital acquired or community acquired, occur in all age groups in both gender. ${ }^{2}$

Indwelling urinary catheters are essential components of modern medical care and are used in acute care setting, extended care facilities, in a person with injured spinal cord, intractable urinary incontinence and urinary retention. Unfortunately indwelling devices significantly increase the risk of iatrogenic Catheter associated urinary tract infection (CAUTI) particularly in an already fragile patient population with potentially pathogenic multidrug resistant organisms which are easily transmitted between patients. ${ }^{3}$ Incidence of UTIs in patients with indwelling catheter is directly related to the duration of catheterization and constitutes the largest institutional reservoir of nosocomial antibiotic resistant organisms. ${ }^{4}$

Catheter associated urinary tract infection(CAUTI) is defined as the new appearance of bacteriuria of predominate bacterial species or funguria of $\geq 10^{2}$ $\mathrm{CFU} / \mathrm{ml}$ in a patient with indwelling catheter of more than 24 hours duration. ${ }^{5}$ CAUTI is most common nosocomial infection accounting for $40 \%$ of all institutionally acquired infections with an incidence of more than 1 million per year. Nosocomial bacteriuria or candiduria develop in up to $25 \%$ of patients requiring a urinary catheter for $\geq 7$ days with daily risk of $5 \% .{ }^{6}$ CAUTI is second most common cause of nosocomial blood stream infection secondary to bacteraemia accounting for $1-4 \%^{3}$ and is most common source of Gram Negative bacteraemia in hospitalized patients. ${ }^{4}$

Biofilm formation on the surfaces of indwelling urinary catheter has important implications for pathogenesis, treatment and prevention of urinary tract infection of CAUTI. ${ }^{3}$ Biofilm consists of multi layered cell clusters embedded in a matrix of extracellular polysaccharide(slime) which facilitates the adherence of microorganisms to biomedical surfaces and protect from host immune system and antimicrobial therapy. ${ }^{7}$ The longer the urinary catheter remains in place, the greater the tendency of these organisms to develop biofilms and results in UTI. ${ }^{8}$

Nosocomial CAUTI is most frequently caused by Escherichia coli, followed by Klebsiella, Enterobacter, Pseudomonas aeruginosa, Enterococci, Staphylococcus aureus, CONS, Candida species. The source of CAUTI may be endogenous that is colonic flora and flora around the perianal or periurethral region or exogenous that is hands of health care personnel, collecting bag or break in drainage system. ${ }^{4}$

Most patients with CAUTI are asymptomatic. Patients who develop nosocomial UTI have more 
prolonged hospitalization than patients without such an infection. ${ }^{3}$ Seriously ill patients are at high risk for UTI related nosocomial bacteraemia and case fatality rate being approximately $13 \% .^{5}$ CAUTI is significantly associated with increased morbidity, mortality-rate being 5\% and health care costs. ${ }^{3}$ Short term catheterization may be associated with fever and acute pyelonephritis whereas long term catheterization may be complicated by catheter obstruction, urinary tract stones and chronic renal failure. ${ }^{5}$

Hence this study is undertaken to identify the causative organisms and know their antibiotic sensitivity, to estimate production of biofilm qualitatively in Escherichia coli. The accurate and early diagnosis is essential to guide the management of catheter related infections.

\section{Materials and Methods}

The present prospective study was included 250 hospitalized patients who catheterized for more than 24 hours duration.

In this study, hospitalized patients who catheterized for more than 24 hours duration were included whereas pregnant women, patients with UTI before catheterization, patients in whom the catheter removed before one day and patients with intermittent catheterization were excluded from the study.

$3 \mathrm{ml}$ of urine sample aspirated with a sterile syringe from the port of the catheter after disinfecting the port with $10 \%$ povidine-Iodine and transported to laboratory without delay. ${ }^{9}$

The urine sample after noting the macroscopic appearance, subjected to wet mount and Gram staining and inoculated on 5\% sheep blood agar, MacConkey agar and CLED media by standard loop method and incubated at $37^{\circ} \mathrm{C}$ for $24-48$ hours aerobically. Specimen was also inoculated into sabouraud agar slope and incubated at room temperature for 7 days and observed for growth. ${ }^{9}$

In positive cultures, a count of $\geq 10^{2} \mathrm{CFU} / \mathrm{ml}$ was taken as significant bacteriuria. ${ }^{5}$ Positive cultures with colony count $<10^{2} \mathrm{CFU} / \mathrm{ml}$ were considered as insignificant and if no growth was observed after 48 hours of incubation, culture were reported as no growth. The isolates with significant colony count were identified by standard laboratory procedures and antibiotic susceptibility testing was done by Kirby Bauer disc diffusion methods as per CLSI guidelines. ${ }^{10}$

Escherichia coli isolates were tested for biofilm production qualitatively by Tube method and Congo Red Agar method. ${ }^{7}$

In Tube method- a loop of isolates were inoculated in $10 \mathrm{ml}$ Trypticase Soy Broth with 1\% glucose (TSB glu) and incubated at $37^{\circ} \mathrm{C}$ for 24 hours, the tubes decanted and washed with phosphate buffer saline (PH 7.3) and dried and dried tubes stained with crystal violet (0.1). Excess stain is removed and tubes were washed with water and then dried in inverted position and observed for biofilm formation. ${ }^{7}$ Biofilm formation was considered positive when a visible film lined the wall and bottom of the tube. In Congo Red Agar method (CRA) - isolates were inoculated on CRA and incubated aerobically for $24-48$ hours at $37^{\circ} \mathrm{C}$. Positive results were indicated by black colonies with dry crystalline consistency. ${ }^{7}$

\section{Results}

In this study of 250 cases, $184(73.6 \%)$ were male and $66(26.4 \%)$ were female. The mean age for males and females was 58.24 years and 56.7 years respectively. The most common age group affected was 51-60 years (20.4\%) followed by $61-70$ years (17.6\%) and $41-50$ years $(17.2 \%)$.

Out of 250 cases, $68(27.2 \%)$ cases had developed significant bacteriuria whereas no growth observed in $182(72.8 \%)$ cases with total percentage of culture positivity being $27.2 \%$.

Out of 68 positive cultures, 65 (95.58\%) were monomicrobial and $3(4.41 \%)$ were polymicrobial and the organisms found in polymicrobial infections were Escherichia coli and Klebsiella pneumonia.

166 patients were with $<6$ days of catheterization, 9 (5.42\%) of them had developed significant bacteriuria and 84 patients were with $\geq 6$ days of catheterization, 59 (70.23\%) of them had developed significant bacteriuria.

In this study, the most common organism isolated was Escherichia coli 24 (33.8\%) followed by Klebsiella spp 15 (21.12\%), Pseudomonas aeruginosa 12 (16.9\%), Enterobacter aerogenes $6(8.45 \%)$, Proteus mirabilis 5 (7.04\%), Staphylococcus aureus $4(5.63 \%)$, Candida albicans $3(4.25 \%)$, Enterococcus faecalis and Citrobacter freundii (1.4\% each).

The most effective antibiotic against Escherichia coli was Imipenem (100\%), followed by Nitrofurantoin (70.83\%), Amikacin, Netilmicin and Tobramycin (58.33\% each). Klebsiella spp showed $93.33 \%$ sensitive to Imipenem, followed by $73.33 \%$ to Nitrofurantoin, $53 \%$ to Amikacin, Netilmicin and Tobramycin. Pseudomonas aeruginosa was $91.61 \%$ sensitive to Imipenem, followed by $66.66 \%$ to PiperacillinTazobactam, $58.33 \%$ to Amikacin, Netilmicin and Tobramycin.

The most effective antibiotic against Staphylococcus aureus was Vancomycin and Linezolid $(100 \%$ each), followed by Gentamicin and Nitrofurantoin (75\% each) and Norfloxacin (50\%) and against Enterococcus faecalis was Vancomycin, Linezolid and Nitrofurantoin.

The total number of Escherichia coli isolates were 24 and $22(91.6 \%)$ of them were biofilm producers. 
Graph 1: Age and gender distribution

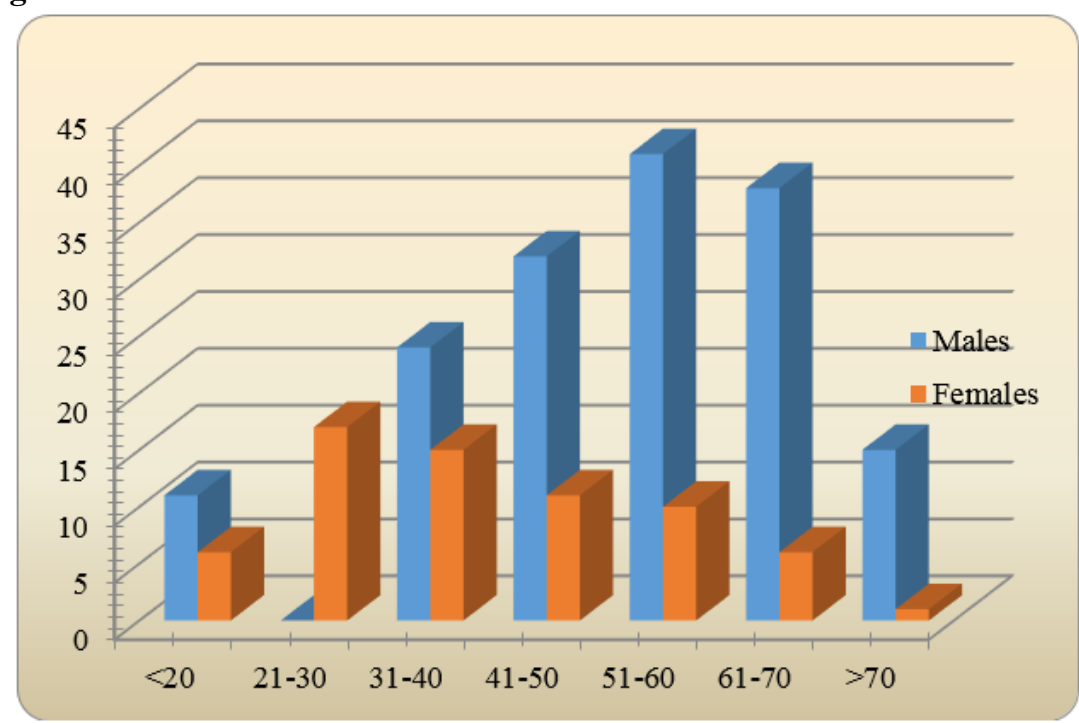

Table 1: Culture results

\begin{tabular}{|l|c|c|}
\hline \multicolumn{1}{|c|}{ Culture results } & No. of cultures & Percentage \\
\hline Significant bacteriuria $\left(\geq 10^{2} \mathrm{CFU} / \mathrm{ml}\right)$ & 68 & $27.2 \%$ \\
\hline No growth & 182 & $72.8 \%$ \\
\hline Total & 250 & $100 \%$ \\
\hline
\end{tabular}

Table 2: Association between duration of catheterization and CAUTI

\begin{tabular}{|l|c|c|c|}
\hline $\begin{array}{c}\text { Duration of } \\
\text { catheterization }\end{array}$ & $\begin{array}{c}\text { No. of patients with } \\
\text { catheterization }\end{array}$ & $\begin{array}{c}\text { No. of patients } \\
\text { with SBU }\end{array}$ & Percentage \\
\hline$<6$ days & 166 & 9 & $5.42 \%$ \\
\hline$\geq 6$ days & 84 & 59 & $70.23 \%$ \\
\hline Total & 250 & 68 & $27.2 \%$ \\
\hline
\end{tabular}

Table 3: Uropathogens isolated in the study group

\begin{tabular}{|l|c|c|}
\hline Isolates & No. of isolates & Percentage \\
\hline Escherichia coli & 24 & $33.80 \%$ \\
\hline $\begin{array}{l}\text { Klebsiella spp } \\
\text { Klebsiella pneumoniae (13) } \\
\text { Klebsiella oxytoca (2) }\end{array}$ & 15 & $21.12 \%$ \\
\hline Pseudomonas aeruginosa & & \\
\hline Enterobacter aerogenes & 12 & $16.90 \%$ \\
\hline Proteus mirabilis & 6 & $8.45 \%$ \\
\hline Staphylococcus aureus & 5 & $7.04 \%$ \\
\hline Candida albicans & 4 & $5.63 \%$ \\
\hline Enterococcus faecalis & 3 & $4.25 \%$ \\
\hline Citrobacter freundii & 1 & $1.40 \%$ \\
\hline Total & 1 & $1.40 \%$ \\
\hline
\end{tabular}

Table 4: Antibiotic susceptibility pattern of Gram negative isolates

\begin{tabular}{|l|c|}
\hline \multicolumn{1}{|c|}{ Antibiotic } & Susceptibility (in Percentage) \\
\hline Imipenem & $95.7 \%$ \\
\hline Nitrofurantoin & $63.4 \%$ \\
\hline Amikacin, Netilmicin and Tobramycin & $58.7 \%$ \\
\hline Amoxicillin-clavulanic acid & $36.5 \%$ \\
\hline Cefotaxime & $33.3 \%$ \\
\hline
\end{tabular}




\begin{tabular}{|l|c|}
\hline Gentamicin & $21.1 \%$ \\
\hline Cefuroxime & $19 \%$ \\
\hline Ciprofloxacin and Norfloxacin & $17.4 \%$ \\
\hline Co-trimoxazole & $7.9 \%$ \\
\hline Ampicillin & $1.5 \%$ \\
\hline
\end{tabular}

Table 5: Antibiotic susceptibility pattern of Gram positive isolates

\begin{tabular}{|l|c|}
\hline \multicolumn{1}{|c|}{ Antibiotic } & Susceptibility (in Percentage) \\
\hline Vancomycin & $100 \%$ \\
\hline Linezolid & $100 \%$ \\
\hline Gentamicin & $75 \%$ \\
\hline Nitrofurantoin & $75 \%$ \\
\hline Norfloxacin & $40 \%$ \\
\hline $\begin{array}{l}\text { Erythromycin, clindamycin, Co- } \\
\text { trimoxazole and Tetracycline }\end{array}$ & $20 \%$ \\
\hline Penicillin & $0 \%$ \\
\hline
\end{tabular}

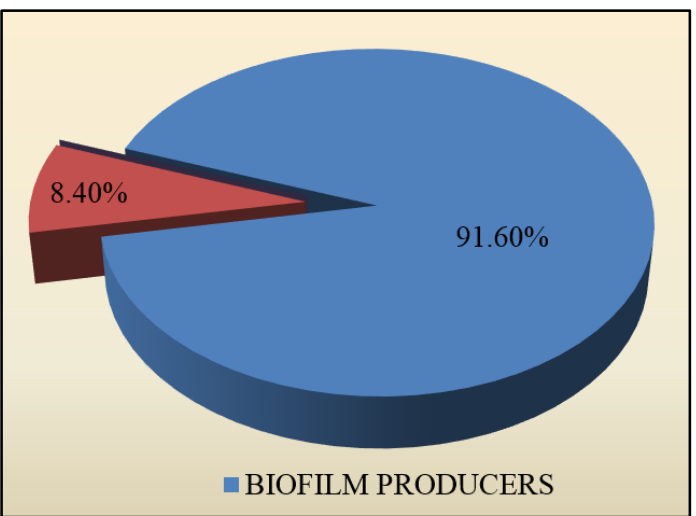

Fig. 1: E.coli isolates producing biofilm

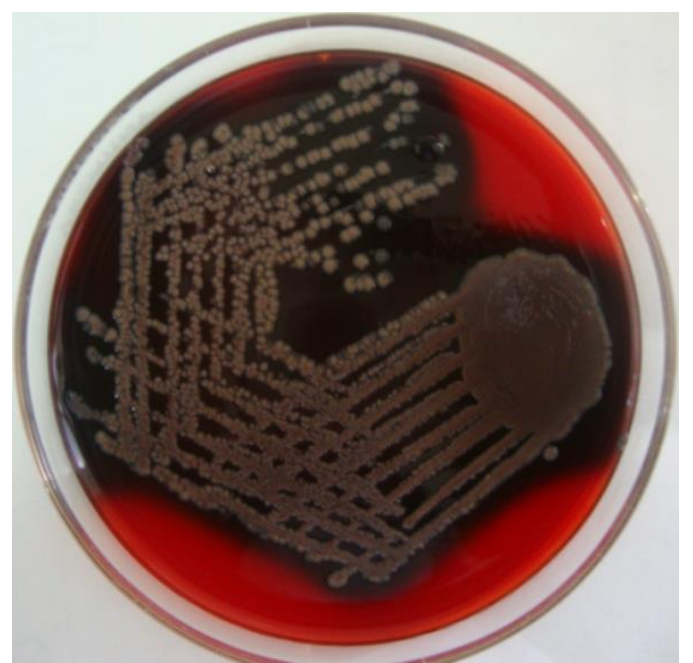

Fig. 2: Biofilm producer forming black colonies with dry crystalline surface on Congo Red Agar

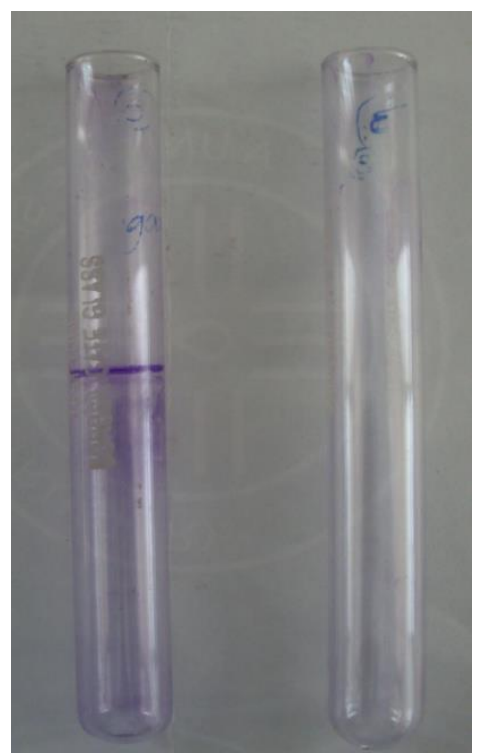

Fig. 3: Tube method; a. Biofilm producer forming thin layer coating the bottom and walls of the tube; $b$. Non biofilm producers forming no layer in the tube

\section{Discussion}

Incidence of UTIs in patients with indwelling catheter is directly related to the duration of catheterization and constitutes the largest institutional reservoir of nosocomial antibiotic resistant organisms. ${ }^{4}$

In this study, incidence of CAUTI was $27.2 \%$. This is in comparison with the studies conducted by Moataz M. Abdul Fallah et al. ${ }^{11}(26.7 \%)$ and Umesh S et al. ${ }^{2}$ $(33.6 \%)$.

The study showed CAUTI was common in the age group of 51-60 years, the mean age being 58 years. This is in comparison with study conducted by Verhaz A et al. ${ }^{12}$ (mean age 60) and Savas L et al. ${ }^{13}$ (mean age 61).

In the present study of 250 cases, females $(28.78 \%)$ were more commonly affected than males $(26.63 \%) \&$ this correlates with studies of Garibaldi et al. ${ }^{14}(32.2 \%)$, Platt R et al. ${ }^{15}(30 \%)$, and Stamm WE et al. ${ }^{6}(32 \%)$ \& this 
difference is attributed to shorter length of female urethra and it's proximity to anal canal.

The study showed $27.2 \%$ samples were culture positive with significant bacteriuria \& this is in comparison with the study of Moataz M. Abdul Fallah et al. ${ }^{11}(26.7 \%)$ and Umesh S et al. ${ }^{2}(33.6 \%)$.

In this study, $95.5 \%$ showed monomicrobial growth and $4.41 \%$ showed polymicrobial growth, is in comparison with the study of Tambyh et al. ${ }^{16}$ (94\% and $65 \%$ ) and Maki DG et al. ${ }^{17}$ (93.58\% and $4.41 \%$ ). The organism isolated in polymicrobial growth were E.coli and Klebsiella pneumoniae, is in comparison with organism isolated in Tambyh et al study. ${ }^{16}$

The study showed $70.23 \%$ of patients with $\geq 6$ days of catheterization and $3.5 \%$ patients with $<6$ days of catheterization developed CAUTI, is in comparison with study of Garibaldi et al. ${ }^{14}(68.25 \%$ \& $3.5 \%)$ and Shapiro et a. ${ }^{18}(72 \% \& 2.65 \%)$.

In this study, Escherichia coli was the most predominant organism isolated (33.8\%) and this finding correlated with Muresul E et al. ${ }^{19}(32 \%)$, Leblebiciogu H et al. ${ }^{20}(32.4 \%)$, and Johansen TE et al. ${ }^{21}(31 \%)$. The next predominant organism isolated was Klebsiella spp $(21.12 \%)$ and this finding correlated with and Custovic A et al. ${ }^{22}(22.6 \%)$. Pseudomonas aeruginosa was the third most common organism isolated (16.9\%) and is in comparison with the study of Leblebiciogu $\mathrm{H}$ et al. ${ }^{20}$ (16.8\%). Enterobacter freundii was the fourth most common organism isolated $(8.45 \%)$ and is in comparison with study of Johansen TE et al. ${ }^{21}$ (6.9\%). Staphylococcus aureus was isolated in $5.63 \%$ of cases and is in comparison with study of Muresu E et al. ${ }^{19}(5 \%)$ and Moataz et al. ${ }^{11}$ (4.7\%). Candida albicans was isolated in $4.25 \%$ of cases and this finding correlated with Muresu E et al. ${ }^{19}(3 \%)$ and Moataz et al. ${ }^{11}(5.8 \%)$. Enterococcus faecalis was isolated in $1.4 \%$ cases, is in comparison with study of Muresu E et al. ${ }^{19}(1 \%)$ and Moataz et al. ${ }^{11}(1.2 \%)$.

In this study, predominant Gram negative organisms isolated were Escherichia coli, Klebsiella spp and Pseudomonas aeruginosa and predominant Gram positive organisms isolated was Staphylococcus aureus $\&$ this is in comparision with other studies.

In general, the most effective antibiotics against Gram negative isolates were Imipenem (95.23\%), Nitrofurantoin (63.49\%), Amikacin, Netilmicin and Tobramycin $(58.73 \%)$. They were least sensitive to Amoxicillin-clavulanic acid (36.5\%), Cefotaxime (33.33\%), Gentamicin (21.12\%), Cefuroxime (19.04\%), Ciprofloxacin and Norfloxacin (17.46\% each), Cotrimoxazole (7.93\%) and Ampicillin (1.58\%) and this is in comparision with other studies.

In general, most effective antibiotic against Gram positive isolates were Vancomycin and Linezolid (100\% each), followed by Gentamicin and Nitrofurantoin (75\% each) and Norfloxacin (40\%). They were least sensitivity to Erythromycin, clindamycin, Co-trimoxazole and
Tetracycline (20\% each) \& this is in comparision with other studies.

In the present study, $91.6 \%$ of Escherichia coli isolates from CAUTI showed significant biofilm production and is in comparison with study of Suman E et al. ${ }^{1}(92 \%)$. There is significant correlation between biofilm production and resistance to multiple antibiotics.

\section{Conclusion}

From the study, it can be concluded that the duration of catheterization is the most important predisposing factor for developing CAUTI. Escherichia coli is the most common organism isolated and high incidence of resistance to conventional antibiotic such as Ampicillin, Co-trimoxazole, Ciprofloxacin, Norfloxacin and gentamicin among the pathogens causing CAUTI. Biofilm production by pathogens is responsible for pathogenesis of UTI and resistance to multiple antibiotics in catheterized patients.

The justified urethral catheterization is the key measure for the prevention of CAUTI and decreasing in CAUTI related morbidity and mortality.

\section{References}

1. Suman E, Jose J, Varghese S, Kotian MS. Study of biofilm production in Escherichia coli causing urinary tract infection. Indian J Med Microbial. 2007;25(3):305306.

2. Umesh S. Kamath, Agnelo Fereirra, Dilip Amonkar, Dilip D Motghare, Manoj S Kulkarni. Epidemiology of hospital acquired urinary tract infection in a medical college hospital in Goa. Indian Journal of Urology. 2009.

3. Barbara W, Traunter, Robih O, Darouiche. Catheter Associated Infections: Pathogenesis Affects Prevention. Arch Intern Med. 2004;164(8):842-850.

4. Campbell' s urology, $8^{\text {th }}$ edition Vol 1 Chapter 10:516519. Philadelphia: Saunders; 2002.

5. Sanjay Saint, Benjamin A, Lipsky. Arch Intern Med. 1999;159:800-808.

6. Stamm WE. Catheter-associated urinary tract infection: Epidemiology, pathogenesis and prevention. Am J Med. 1991;91:65S-71S.

7. Mathur T, Singhal S, khan S, Upadhyay DJ, Fatima T, Rattan A. Detection of biofilm formation among the clinical isolates of Escherichia coli: An evaluation of three different screening methods. Indian $\mathrm{J} \mathrm{Med}$ Microbiol. 2006;24:25-29.

8. Rodney M. Donlan. Biofilm and Device-Associated infections. CDC.2001;7(1)

9. Paul A. Tambyah, Dennis G. Maki. The relationship between pyuria and infection in patients with indwelling urinary catheters. Arch Intern Med. 2000;160:673-677.

10. Collee JG, Fraser AG, Marmion BP, Simmons A. Mackie and McCartney - Practical Medical Microbiology. $14^{\text {th }}$ ed. Edinburg: Churchill Livingstone; 1996.

11. Moataz M. Abdul-Fattah. Surveillance of nosocomial infections at a Saudi Arabian military hospital for a oneyear period. GMS German Medical Science. 2005;3:1612-14.

12. Verhaz A, Skrbic R, Rakita-Music M. Resistance of catheter-associated urinary tract infection to antibacterials. Vojnosanit Pregl. 2005;62(3):181-7. 
13. Savas L, Guvel S, Onlen Y, Savas N, Duran N. Nosocomial urinary tract infection: micro-organisms, antibiotic sensitivities and risk factors. West Indian Med J. 2006;55(3):188-93.

14. Garibaldi RA, Burke JP, Dickman ML, Smith CB. Factors predisposing to bacteriuria during indwelling urethral catheterization. N Engl J Med.1980; 291:215219.

15. Platt R, Polk BF, Murdock B, Rosner B. Risk factors for nosocomial urinary tract infection. Am J Epidemiol. 1986;124:977-985.

16. Paul A. Tambyah, Dennis G. Maki. The relationship between pyuria and infection in patients with indwelling urinary catheters. Arch Intern Med. 2000;160:673-677.

17. Stark RP, Maki DG. Bacteriuria in the catheterized patients. What quantitative level of bacteriuria is relevant? N Engl J Med. 1984;311:560-564.

18. Shapiro M, Simchen E, Izraeli S, Sacks TO. A multivariate analysis of risk factors for acquiring bacteriuria in patients with indwelling urinary catheters for longer than 24 hours. Infect Control. 1984;5:525-532.
19. Muresu E, Pechmann A, Manaca E, Cappal M, Demontis LM, Valca D. Urinary tract infections: observation on 19,021 patients hospitalized in Sassari Province. Quad Sclavo Diagn.1986;22(4):367-87.

20. Leblebiciogu H, Esen S. Turkish Nosocomial Urinary tract infection in Turkey. J Hosp Infect. 2003;53(3):20710.

21. Johansen TE, Cek M, Naber KG, Stratchounski L, Svendsen MV, Tenke P. Int J Antimicrob Agent. 2006;28(1):S91-107.

22. Custovac A, Zulcic-Nakic V, Asceric M, Hadzic S. Surveillance of intrahospital infections. Bosn J Basic Med Sci. 2009;9(1):66-70.

How to cite this article: Vidyasagar $K$, Nagarathnamma T. Study of catheter associated urinary tract infection and biofilm production by Escherichia coli. Indian $\mathbf{J}$ Microbiol Res. 2018;5(3):387-392. 